\title{
Optimal Time Allocation between Idle and Active Time
}

\author{
Michal Weber ${ }^{1}$, Limor D. Gonen ${ }^{2}$, Sara Westreich ${ }^{3}$, Uriel Spiegel ${ }^{4}$ \\ ${ }^{1}$ Faculty of Business Administration, Ono Academic College, and Department of Management, Bar-Ilan University, \\ Israel \\ ${ }^{2}$ Department of Economics and Business Administration, Ariel University, Israel \\ ${ }^{3}$ Department of Management, Bar-Ilan University, Israel \\ ${ }^{4}$ Department of Management, Bar-Ilan University, Israel and Zefat College, Israel \\ Correspondence: Limor Dina Gonen, Department of Economics and Business Administration, Ariel University, Israel.
}

\author{
Received: May 30, $2017 \quad$ Accepted: June 20, $2017 \quad$ Available online: June 21, 2017 \\ doi:10.11114/aef.v4i4.2506 URL: https://doi.org/10.11114/aef.v4i4.2506
}

\begin{abstract}
Idle time is an essential and valuable factor in the production of any service. While idle time is necessary and helpful for efficient and effective utilization of time, it also has a negative effect that managers try to minimize. This paper illustrates either analytically or numerically the different effects of idle time on total net revenue. It first presents the case in which idle time is determined arbitrarily, and then shows idle time that has no direct negative effect on total revenue but a positive effect on the efficient use of active time.
\end{abstract}

Keywords: idle time, productive active time, time management, interdependency between idle and active times, total revenue

\section{Introduction}

Idle time is a fundamental and valuable factor in the creation or production of any service. It is inevitable today in the routine process of the economy. The Biblical commandment established the Sabbath day of rest from the time of the creation of the world. That day is interpreted and translated as "idle time" for resting after six working days. Various societies later extended the idle time to last for two consecutive days of rest that immediately follow five days of work, as in most of the Western world today.

Neuroscientists have discovered new evidence revealing the importance of idle time for the routine activity of the brain during active time. The uniqueness of idle time has become an issue investigated in recent years by prominent scientists, especially in neuroscience research. Several recent studies are referenced below.

"The more we study the brain and evolution, the more we're discovering that the idle brain is more likely to come up with new ideas" (Innovation Tip: Schedule Idle Time, future think weblog-see https://futurethink.wordpress.com/2010/08/05/innovation-tip-schedule-idle-time). The blog asserts that an individual who seeks creative and original thinking should regularly incorporate idle time into his schedule. It further explains that idle time means mental breaks that exclude activities such as research, meetings, or email communications.

This concept is also discussed in the blog "Your Brain Unplugged: Proof That Spacing Out Makes You More Effective," that is based on an interview with Andrew Smart, author of "Autopilot: The Art \& Science of Doing Nothing" (Ursrey, 2014), It is further summarized below.

When individuals undertake too many tasks, they actually are less efficient. It has been scientifically proven that idle time is required for developing creativity. The Default Mode Network of the brain becomes better focused during idleness and the brain may even exert more effort while a person remains idle. Neuroscience has shown that idleness is required for reaching one's potential. The brain's need for idleness may also be compared to the need for enough sleep. When a deficit builds up, it has a negative effect on how an individual functions. Idleness is a basic human right and instinct. Leisure that is practiced with awareness and beneficial for one's health has decreased over time. In contrast, resting remains a natural instinct for all animals. If one remains aware of the scientifically proven importance of idleness for better health and lives accordingly, brain function will improve, time will seem to pass more slowly, and an individual will be capable of increasing his accomplishments. 
Idle time can be broken down further, not in relation to a week but even as a portion of each day. Part of the 24-hour day can be devoted to labor and the remaining time to leisure activities such as sleeping, reading, watching TV, playing with children and grandchildren, and other kinds of entertainment, or as simply stated, idle time. The working hours of the day also include idleness. During daily hours of work the mechanic who fixes a car or the professor who works on research each leaves his workplace and computer for breakfast, lunch, or a short coffee break. Thus both activity and idle time are required and necessary within working hours.

During writing or reading, other important and required idle times are due to personal needs such as coffee breaks, lunchtime, and rest, all of which enable efficient activity time. We refer to this sort of idle time as positive and desired idle time. For example, athletic training requires idle time for resting, drinking, eating, etc. between exercises and other physical efforts.

As discussed above, idle time is a positive and important factor, required and helpful for using time efficiently and effectively. However, idle time can also have a negative effect that managers seek to eliminate or to minimize. Every working hour can be divided even further. Some portion of working hours is bound to be wasted. In part, idle time is even required for work reasons such as in receiving assignments for the following day, performing machinery adjustments, waiting between completing an earlier job and beginning a new one, waiting for instructions concerning a new task, or supplying material, tools, etc. Idle time that is undesirable or wasteful should decrease and this is a specialization in operations research. Such idle time is also referred to as unutilized idle time that should be minimized, and it is not included in the category of idle time in the present paper. The following illustrates unutilized idle time.

Sometimes such "unproductive" time results from factors beyond an employee's control. Unutilized idle time is the time associated with waiting or with underutilization of available machinery. As demonstrated in the preceding examples, time management is extremely important in any business or active employment process. This includes coordinating tasks that are dependent upon one another. For example, when one task requires that another first be completed elsewhere, the two should be synchronized in order to enable a smooth transition between them and decrease unutilized idle time. This kind of unplanned idle time may result from a deficiency in a production process. The present analysis, however, is different. Sometimes idle time is a desirable, planned and necessary part of an efficient process. However, while idle time is required in the production process, it nevertheless should be managed efficiently.

The present paper deals with time management that includes willingly and voluntarily initiating idle time for the sake of more efficient and effective use of the active time that follows.

Idle time can be categorized according to functional causes including among them: (a) production causes such as lack of materials, issues related to repair and maintenance of machinery, unutilized human resources, prior operations, etc.; (b) administrative causes such as insufficient planning, delayed work instructions, etc.; or (c) economic causes such as lack of product demand requiring the partial use of machinery; work stoppage resulting from strikes, lock-outs etc. (Acharya). All of these three categories represent unplanned situations of idle time that are imposed on the system. They represent deficiencies that may occur during the production process. The present paper, however, seeks to deal with idle time that is planned and necessary for efficient normal activity.

Idle time that represents deficiency requires devices to lower their costs. One such example is the reduction of energy consumption as described below.

In recent years, concern has increased with respect to wasted energy consumption in computing infrastructure. Networked end-systems frequently remain powered-on although they are idle, thus resulting in wasted energy consumption. However, power management options now enable the reduction of computer power consumption. Such techniques are based upon hardware support for sleep states so that during idle times the power consumption of various sub-components can be decreased to different levels (Nedevschi, et al., 2009).

Although the World Wide Web (WWW) is important for electronic information retrieval, commerce and entertainment, long download times have remained very problematic for Web users (Selvidge, 1999, 2003), Studies by Lightner, Bose and Salvendy (1996) and the Graphic, Visualization and Usability (GVU) Centre at Georgia Institute of Technology (GVU, 1998), indicate that lengthy download times have always been a significant difficulty for Web users. Pitkow and Kehoe (1996), also show that the most common complaint with using the WWW, as indicated by $69 \%$ of respondents, is the amount of time required to download Web pages. This problem has become worse over time due to the exponential increase in Web users and the popularity of multimedia technology.

\subsection{Unutilized Idle Time Necessitates - Saving Car Energy}

Idle time is the period in which a vehicle's engine is running, but the vehicle is not traveling. Vehicles usually idle in heavy traffic or for longer periods of time when used for deliveries. While idling in traffic a vehicle might burn a quarter of its fuel and emit a quarter of its $\mathrm{CO} 2$, thereby wasting energy and exacerbating climate change. Included among the benefits of decreasing idle time are lower costs of engine maintenance, longer engine life, decreased 
emissions of toxic air pollutants and carbon dioxide, less noise and pollution, rand educed dependency on oil imports. These issues have resulted in the development of vehicles such as hybrid cars which use dual energy sources.

All of these preceding examples illustrate the existence of unutilized idle time for which devices are sought to eliminate or minimize its undesirable effect.

This paper deals with time management that concerns the willing and voluntary initiation of idle time for the sake of more efficient and effective use of subsequent active time.

The following discussion illustrates the requirement for positive and desirable idle time that brings greater efficiency to various kinds of activities in diverse areas of life.

For example, a farmer grows different kinds of fruit and vegetables during a period of several months or years, which is divided into a number of cycles of specified duration. Each cycle contains required active time for watering the crops, followed by a specified idle time. At certain times the farmer waters the plants (or trees) allowing diminishing productivity of units of water on the crops. The watering is followed by idle time and then fertilization, spraying, and weeding. These activities also require planned idle time before resuming the watering and so forth, in a repeating cycle. Without idle time endless irrigation or spraying might lead to flooding and damage or to burning the plants rather than growing them efficiently. Sometimes even the cycle activities of irrigation as well as fertilizing or spraying require idle time within them that also becomes part of the cycle. Decreasing or completely eliminating idle time disturbs the normal and efficient process of growing the fruits or the vegetables. Finally, it is not known whether the farmer maintains cycles of land use. The land should rest between cycles of plowing and sowing in order to grow better crops. It is well known that land abuse is not desirable.

An additional example concerns diverse complementary therapies for improving health through relaxation. Some use relaxation for enabling psychological change. Others include movement, stretching, and breathing exercises. Hypnosis, relaxation, and stress management are sometimes also used in conventional medicine. Yoga and meditation practices are very accessible and often related to fitness and wellbeing. They are also increasingly used for relieving symptoms in chronic and life-limiting illnesses.

Yoga practice utilizes postures, breathing exercises, and meditation focused on enhancing mental and physical functioning. Sometimes yoga is regarded as physical exercise and mental relaxation.

\subsection{Examples of the Importance of Idle Time - Medicine}

The most commonly used first line drug for malaria is chloroquine. Since resistance to chloroquine is now widespread, a new first line drug should be adopted. The decision regarding the optimal time for the switch in the line drug is complex and involves trade-offs in costs and health outcomes while many parameters are uncertain. It depends on objective values such as initial resistance and resistance growth rates, and on subjective values such as bureaucracy and time preferences of policymakers (Goodman, Coleman, \& Mills 2001; Coleman, Morel, Shillcutt, Goodman, \& Mills 2004).

The rapidly growing global problem of resistance to antimicrobial agents used to treat common infectious diseases is severe for malaria (World Health Organization [WHO], 1996). The resistance to antimalarial treatment is significantly increasing the burden of disease. The uncomplicated malaria symptoms include chills, nausea and fever. If not effectively treated, severe malaria may include symptoms such as convulsions, coma and severe acidosis, and a high fatality rate. Access to prompt and effective treatment would substantially reduce malaria, but the development of drug resistance hampers the capacity of health services to provide adequate care.

A key question for policymakers is the level of resistance at which a change in drug policy should be implemented (Bloland et al., 1998; Sudre, Breman, McFarland, \& Koplan 1992; Bloland et al., 1993; Schapira Beales, \& Halloran 1993). Analysis of this issue involves consideration of both the costs and health effects of changing drugs.

Changing a line drug leads to increased costs of new drugs. Costs associated with the policy change involve revising treatment guidelines, training staff and producing new health education materials. Cost savings (cost offsets) may arise if the new regimen is more effective, thereby reducing the number of persistent cases of uncomplicated malaria and the number of cases developing into severe malaria. In order to incorporate the trade-offs in costs and health outcomes it is necessary to evaluate the new treatment over time (Goodman, Coleman, \& Mills 2001; Coleman, Morel, Shillcutt, Goodman, \& Mills, 2004).

The contrasting reluctance of national policymakers in Africa to change first line antimalarial drugs, even in the face of high levels of drug resistance, can be attributed to a range of factors (Bloland, Ettling, \& Meek 2000). First, good data are lacking with regard to costs and health outcomes. Second, national policymakers may implicitly use a longer timeframe if they lack confidence in the future availability of effective and affordable alternatives. Finally, changing first line antimalarial policy is a high profile and politically sensitive decision, so policymakers need a high degree of 
confidence that it is appropriate to recommend change.

\subsubsection{Depression Medication}

Half of individuals with depression require medication other than the antidepressant originally prescribed for them (Garcia-Toro et al, 2012; Connolly \& Thase, 2011; Trivedi et al., 2009; Papakostas, 2009; Souery, Serretti, \& Calati 2011; Fredman et al., 2000). In addition, half of those who switch to a new medication might still require a different antidepressant (Connolly \& Thase, 2011). Determining the correct treatment often necessitates trying alternative medications.

When patients do not respond to a specific antidepressant or when they experience side effects, another antidepressant is needed. The physician must have knowledge concerning the drug that is being replaced, possible interactions between drugs, and the time in which the replacement medication becomes effective. In addition, when antidepressants are discontinued an antidepressant withdrawal syndrome must be avoided (Hirsch \& Birnbaum, 2012; Ogle \& Akkerman, 2013).

Hirsch and Birnbaum (2012) present the techniques described below for switching drugs.

Cross-tapering - Cross-tapering is often the method that best assures that depression is not unmasked by rapid drug withdrawal, while it also minimizes the risk of drug interactions. The dose of an antidepressant is gradually decreased over at least one to two weeks, while another antidepressant is initiated and gradually increased to therapeutic range.

Switching between SSRIs - This is generally the simplest antidepressant switch. Selective serotonin reuptake inhibitors (SSRIs) overlap in their mechanism of action, so that the new SSRI usually prevents discontinuation symptoms. A new SSRI can usually be substituted at a relatively equivalent dose of a former SSRI (Table 1). However, the new SSRI may also be initiated at a lower dose since patients sometimes experience idiosyncratic side effects to certain SSRIs.

Switching from SSRI to TCA - The switch from an SSRI to a tricyclic antidepressant (TCA) most often uses a cross-taper method (see Cross-tapering, above). Fluoxetine and paroxetine are much stronger inhibitors of the p450 enzyme 2D6 than are sertraline, citalopram, and escitalopram. This enzyme is involved in the metabolism of many TCAs and inhibition increases TCA blood level, which can result in toxicity. Therefore, TCAs should be first given at low doses when cross-tampering with an SSRI, especially with fluoxetine and paroxetine. TCA blood levels can be tested at this time as a further precautionary measure.

\subsubsection{Cancer Treatments}

Chemotherapy involves various considerations, including among them the type of cancer, its location, whether and where it has spread, and the individual's general health. The course of chemotherapy is given according to a treatment plan. It usually consists of several cycles of chemotherapy that are separated by rest periods. The rest periods enable recovery of healthy and normal cells from any undesirable effects of the drug. Although the drugs are intended to destroy tumor cells, they also destroy normal cells. Thus a chemotherapeutic protocol must consider both how well the tumor is destroyed and how normal cells are best protected. A successful chemotherapy cycle depends upon determining the duration of treatment and recovery periods. The timing is extremely important (Zietz, Desaire, Grattarola, \& Nicolini 1978; Swierniak, Kimmel, \& Smieja, 2009).

The end of this section provides a few more examples in which positive idle time is helpful for efficient active time in learning. The learning process at high school or in academic institutions of higher education requires certain hours of active learning in class lectures as well as hours of practice. The teacher needs to determine how often or how long to teach a course as well as the extent of the idle time in between class meetings. Should the course be taught in one long week of 26 hours or throughout an entire semester for 2 hours each week during 13 weeks? The optimal idle time in relation to the active time is an important issue for further investigation.

A final case discussed in the transportation literature further illustrates the preceding discussion. It is assumes, for instance, that truck drivers drive for T hours annually. For every cycle of $\hat{t}$ hours the law requires idle time of $t_{I}$. The questions to be determined are the number of hours of active driving, $t_{A}$, that correspond to the required idle time; and the optimal length of $\hat{t}=t_{A}+t_{I}$ for each cycle.

The next section develops a mathematical model in which the time allocation for a given cycle is divided between active time and idle time for several kinds of production functions of time.

Idle time has been recognized by scientists in different disciplines as a positive and important factor in productive and active time. A model has been developed regarding this issue and today the phenomenon of the positive intertemporal effect of idle time on active time is acceptable. The models presented below seek to reflect different functions with different kinds of interdependency that can be applied to the actual cases mentioned earlier.

Paradoxically, doing and achieving more may be accomplished by relaxing and exerting less effort. The worker, the 
manager, and the economic planner should recognize that idle time is not undesirable but something that is welcome. In accordance with this new approach, what appears to be nonproductive time that ostensibly brings inefficiency to production and active time is instead a crucial and important factor for greater efficiency and productivity.

\section{Theoretical Model}

\subsection{Optimal Active Time for the Case of a Given Exogenous Idle Time}

When idle time is arbitrarily determined either by religion or a policymaker, the manager has to determine the optimal active time $t_{A}$ for the given and required value of $t_{1}$.

According to the Bible, each week the Sabbath day of rest follows six days of work. The day of rest was also required by law in modern Western countries. However, in most of these countries policymakers later shortened the work week to only five days and increased the idle time to two days at the end of the week. This basic social law has been justified by the idea that resting and fulfilling family and social obligations during the weekend may positively affect two elements, the wellbeing of workers who benefit from rest; and their high productivity during the active days of work, $t_{A}$. That high level of productivity is due to the mandatory $t_{l}$.

Assuming a given $t_{I}$ (that is arbitrarily determined at this stage of the analysis) the following function of the marginal revenue of productivity of $t_{A}$, would be $V M P_{t_{A}}$.

We assume that $V M P_{t_{A}}$ is diminishing while $t_{A}$ is increasing by a constant rate as follows:

$$
V M P_{t_{A}}=A-\alpha t_{A}
$$

This indicates that we can define the length of each cycle, $L$, as follows:

$$
t_{A}+t_{I}=l
$$

For a given $t_{I}$ the length of each cycle depends on $t_{A}$ that also affects the total revenue from each active time $t_{A}$ at each cycle, $v$.

$$
v=\int_{0}^{t_{A}} V M P_{t_{A}} d t_{A}
$$

Since we assume at (1) that $V M P_{t_{A}}$ is positive and linearly declines with $t_{A}$ we can rewrite (3) as follows:

$$
v=\frac{\left(A+A-\alpha t_{A}\right) t_{A}}{2}=A t_{A}-\frac{\alpha}{2} t_{A}^{2}
$$

At a given time $\bar{T}$ we find number of cycles, $n$, as follows:

$$
n=\frac{\bar{T}}{t_{A}+t_{I}}
$$

Thus after $n$ identical cycles of revenue $v$ at each cycle we gain total revenue $V$ that should be maximized with respect to $t_{A}$ as follows:

$$
\operatorname{Max}_{t_{A}} V=v \times n=\left(A t_{A}-\frac{\alpha}{2} t_{A}^{2}\right)\left(\frac{\bar{T}}{t_{A}+t_{l}}\right)
$$

Taking the F.O.C. derivative of (5) with respect to $t_{A}$ since $t_{I}$ is given:

$$
\frac{d V}{d t_{A}}=\left(A-\alpha t_{A}\right)\left(\frac{\bar{T}}{t_{A}+t_{I}}\right)-\frac{\bar{T}}{\left(t_{A}+t_{I}\right)^{2}}\left(A t_{A}-\frac{\alpha}{2} t_{A}^{2}\right)=0
$$

From (6) we get (7):

$$
\left(A-\alpha t_{A}\right)\left(\frac{\bar{\tau}}{t_{A}+t_{\bar{I}}}\right)=\frac{\bar{\tau}}{\left(t_{A}+t_{I}\right)^{2}}\left(A t_{A}-\frac{\alpha}{2} t_{A}^{2}\right)
$$

or

$$
\left(A-\alpha t_{A}\right)\left(t_{A}+t_{I}\right)=A t_{A}-\frac{\alpha}{2} t_{A}^{2}
$$

or

$$
A t_{A}+A t_{l}-\alpha t_{A}^{2}-\alpha t_{A} t_{l}=A t_{A}-\frac{\alpha}{2} t_{A}^{2}
$$

From (7") we get a quadratic equation of: 


$$
\frac{\alpha}{2} t_{A}^{2}+\alpha t_{I} t_{A}-A t_{I}=0
$$

that leads to the optimal $t_{A}$ for any given idle time of $t_{1}$ :

$$
t_{A}=\frac{-\alpha t_{I}+\sqrt{\alpha^{2} t_{I}^{\frac{2}{2}+2 \alpha A t_{I}}}}{\alpha}
$$

For simplicity, in order to demonstrate the result at (5) we get the following:

For $t_{I}=1$ for $A=24$ and $\alpha=1$, we get the value of $t_{A}=6$.

For any weekly Sabbath day of rest, people will choose six working days and vice versa. After productive activities during six working days, individuals should take an interval of one day off for $\bar{T}=365$ days a year and $n=52$ weeks.

The questions raised in the previous case are : (i) For a given level of $t_{I}$ what should be the optimal active time? and (ii) For a certain time period of $\bar{T}$, how may cycles, $n$, of idle and active time would be the ideal? Stated otherwise, what is the length of each cycle, $l$, that is equal to $t_{A}+t_{I}\left(\hat{t}=t_{A}+t_{I}\right)$ ?

\subsection{Active and Idle Times are Determined Simultaneously}

The next question is how to determine the $t_{l}$ and $t_{A}$ ideal bundle $t$ since $t_{l}$ was previously determined arbitrarily, and $t_{A}$ was a function of $t_{I}$.

In the cases presented below, we will discuss below with the possibility that both $t_{I}$ and $t_{A}$ are decision variables determined simultaneously in order to determine optimal revenues and find efficient allocation of time between active and idle times. The variable $t_{I}$ is not determined arbitrarily as a parameter, but as a decision variable in addition to $t_{A}$.

The analysis below may also result in the optimal solution of the length and number of the cycles, as well as the optimal proportion ratio at each cycle between $t_{I}$ and $t_{A}$.

By determining the optimal values of $t_{I}$ and $t_{A}$, we determine the length of each cycle, $\hat{t}$. Thus, for a given period $\bar{T}$ we find the number of identical cycles, $n$.

For this last statement, we illustrate either analytically or numerically different effects on the total net revenue, as follows:

A) Idle time has no direct negative effect on the total revenue but it has a positive effect on the efficiency of active time. Thus at idle time there are no direct revenues benefits. However, there are no losses or costs of idle time on revenues.

B) Idle time has a negative effect that maintains a constant marginal cost on the total revenue. This means that during idle time, a constant cost per idle time maintains a constant marginal cost of idle time.

C) Idle time cost and the marginal cost are increasing by a constant rate at each additional unit of idle time. This case is not resolvable analytically and remains a subject for future research.

In the cases discussed below we assume a positive intertemporal effect of idle time that positively affects the efficiency of active time in generating revenues. This is based on the presumption that idle time is required, for example, for the efficiency of the brain during active time. See the blog, "Your Brain Unplugged: Proof That Spacing Out Makes You More Effective" as referenced above. Another possible example is in the case of a truck driver that needs to be alert, etc. before continuing to drive on a highway. A third example is the use of a new drug that is more efficient due to the prior use of another drug.

We begin with case A:

\section{A) The Case in which Idle Time has no Direct Negative Effect on the Total Revenue}

This case assumes a specific shape regarding the value of the marginal productivity of active time $t_{A}$ :

$$
V M P_{t_{A}}=A+B t_{I}^{\beta}-C t_{I}^{\delta} t_{A}^{\gamma}
$$

This case indicates that a specific positive interdependency between idle time, $t_{1}$ and the marginal productivity of active time, $t_{A}$ holds. The effect of $t_{I}$ is positive on the intercept of the parameter, $A$, but also causes an increase of the slope of the diminishing marginal curve, of $V M P_{t_{A}}$. The latter is diminishing faster at higher values of $t_{I}$.

As a result, the total value of revenue of each cycle that is defined as $v$ becomes:

$$
v=A t_{A}+B t_{I}^{\beta} t_{A}-\frac{C t_{I}^{\delta} t_{A}^{\gamma+1}}{\gamma+1}
$$


While the number of cycles, $n$, during period $\bar{T}$ is as mention in equation (4) $n=\frac{T}{t_{A}+t_{I}}$.

And since the total revenue of all cycles $V$ is defined as . $n$, we define the objective function as follows:

$$
\operatorname{Max} V=v \times n=\left(A t_{A}+B t_{I}^{\beta} t_{A}-\frac{c t_{I}^{\delta} t_{A}^{\gamma+1}}{\gamma+1}\right)\left(\frac{\bar{T}}{t_{A}+t_{I}}\right)
$$

We cannot solve this general equation analytically, thus we focus on special cases with special values that allow us to resolve as much as possible analytically. Some of them yield analytical solutions, while for others we can at least present some analytical relationship between $t_{A}$ and $t_{I}$, though without specific optimal values of these last variables.

Example A1:

An analytical solution of optimal idle and active time exists:

Let's assume the following specific values of $\beta=1, \gamma=1, \delta=0$. In this case, the idle time has linear impacts on $\mathrm{v}$ at (12), and the active time has linear impacts on the diminishing marginal productivity. Thus, at equation (13) we start with a very simple structure of $\mathrm{v}$ introduced at equation (3), that depends on two independent inputs: idle time, $t_{l}$, and active time, $t_{A}$, with simple interdependency between $t_{I}$ and $t_{A}$.

Under the above assumptions, we define $\mathrm{v}$ as:

$$
v=A t_{A}+B t_{I} t_{A}-\frac{c}{2} t_{A}^{2}
$$

Where A, B and $\underset{T}{\mathrm{C}}$ are parameters. The total time $\bar{T}$ is allocated for $t_{I}$ and $t_{A}$ with $\mathrm{n}$ identical cycles where (4) still holds, i.e., $n=\frac{T}{t_{I}+t_{A}}$.

Therefore, the total value of accumulated knowledge, $\mathrm{V}$, is equal to $n$.

Thus, (13) becomes:

$$
V=\left[B t_{I} t_{A}+A t_{A}-\frac{C}{2} t_{A}^{2}\right]\left[\frac{\bar{T}}{\bar{t}_{I}+t_{A}}\right]
$$

The two decision variables in this case are, $t_{I}$ and $t_{A}$.

The First Order Condition (F.O.C.) are:

$$
\frac{\partial V}{\partial t_{I}}=B t_{A} \times \frac{\bar{\tau}}{t_{I}+t_{A}}-\frac{\bar{\tau}}{\left(t_{I}+t_{A}\right)^{2}} \times v=0
$$

and

$$
\frac{\partial V}{\partial t_{A}}=\left[B t_{I}+A-C t_{A}\right]\left[\frac{\bar{T}}{t_{I}+t_{A}}\right]-\frac{\bar{T}}{\left(t_{I}+t_{A}\right)^{2}} \times v=0
$$

From (14) and (15) we get:

$$
B t_{A}=B t_{L}+A-C t_{A}
$$

or

$$
t_{I}=\left(\frac{C+E}{B}\right) \cdot t_{A}-\frac{A}{B}
$$

From equation (14), we get the optimal values of active and idle times:

$$
t_{A}^{*}=\frac{2 A}{2 E+C}
$$

From (15) and (16) we get:

$$
t_{I}^{*}=\frac{A C}{B\left(2 B^{*}+C\right)}
$$

For the above optimal values of $t_{I}^{*}$ and $t_{A}^{*}$ at (17) and (18) we illustrate the results for specific parameter values when $\mathrm{A}=42, \mathrm{~B}=6$ and $\mathrm{C}=2 t_{A}^{*}=6$ and $t_{l}^{*}=1$.

We get the specific optimal structure of the seven-day week during the Biblical creation of the world. Today a cycle of seven days in each week includes six days of work followed by one day of rest.. However, due to technological change of the values that affect the value of $t_{I}$ and $t_{A}$, the seven days of the week are split differently. For example if $\mathrm{A}=42$ and $\mathrm{B}=6$ as before, but $\mathrm{C}$ increases and is equal to $\mathrm{C}=4.8$, then the optimal distribution of the seven days of each 
working week would be divided into 5 working days followed by 2 days of rest, as in many Western developed countries today.

Another example is the allocation of the twenty-four hours of a day to 8 hours of work and sixteen hours of leisure that are used for sleeping, learning, watching television, and other idle hours each day: if $A=24, C=2$ and $B=1$, we get optimal active time of $t_{A}^{*}=8$ and idle hours of $t_{I}^{*}=16$. Thus $\hat{t}=24$ represents the total daily hours, that are split in a ratio of $1: 2$.

The discussion below adds several numerical examples since we cannot provide a precise analytical solution, yet sometimes we can analytically express the relationship between $t_{A}$ and $t_{l}$.

Example A2(i):

In this case we determine $\gamma=1$, and $\mathrm{A}=0$ at equation (10).

In this case, the positive value of idle time is necessary for the process. Otherwise, if there is no idle time, there is no positive marginal productivity at all for the active time.

In this case the value of $V M P_{L A}$ is:

$$
V M P_{L_{A}}=B t_{I}^{\beta}-C t_{I}^{\delta} t_{A}
$$

As a result, the total value of revenue of each cycle is $\mathrm{v}$.

$$
v=\frac{\left(2 E t_{I}^{\beta}-C t_{l}^{8} t_{A}\right) t_{A}}{2}
$$

While the number of cycles, $n$, along period $\bar{T}$ is again $n=\frac{{ }^{2}}{t_{A}+t_{I}}$.

Since $V=n \times v$, we define the objective function:

$$
\operatorname{Max}_{t_{l}, t_{A}} V=v \times n=\frac{\left(2 E t_{I}^{\beta}-C t_{I}^{\delta} t_{A}\right) t_{A}}{z}\left(\frac{\bar{T}}{t_{A}+t_{l}}\right)
$$

The F.O.C. with respect to each of the two decision variables is:

$$
\begin{gathered}
\frac{\partial V}{\partial t_{A}}=\left[B t_{I}^{\beta}-C t_{I}^{\delta} t_{A}\right]\left[\frac{\bar{T}}{\bar{t}_{I}+t_{A}}\right]-\frac{\bar{T}}{\left\langle t_{I}+t_{A}\right\rangle^{2}} \times v=0 \\
\frac{\partial V}{\partial t_{I}}=\left(B \beta t_{I}^{\beta-1} t_{A}-\frac{\delta C t_{I}^{\delta-1} t_{A}^{\bar{\tau}}}{2}\right) \frac{\bar{T}}{t_{A}+t_{I}}-\frac{\bar{T}}{\left\langle t_{A}+t_{I}\right\rangle^{2}} \times v=0
\end{gathered}
$$

By comparing (22) and (23) we can express $t_{A}$ in terms of $t_{I}$ as follows:

$$
t_{A}=\frac{E \beta t_{l}^{\beta}+C t_{I}^{\delta+1}-\sqrt{E^{2} t_{l}^{2 \beta} \beta^{2}-2 E C t_{l}^{\delta+\beta+1} \delta+2 E C t_{I}^{\delta+\beta+1} \beta+C^{2} t_{l}^{2(\delta+1)}}}{C t_{l}^{\delta} \delta}
$$

\begin{tabular}{|c|c|c|c|c|}
\hline$\beta$ & $t_{A}$ & $t_{I}$ & $\mathrm{n}$ & $\mathrm{V}$ \\
\hline 0.1 & 5.281 & 0.352 & 64.78 & 6963.3 \\
\hline 0.3 & 6.668 & 1.818 & 43 & 6782.2 \\
\hline 0.5 & $y .604$ & 6.86 & 22.16 & 9447.87 \\
\hline 0.7 & $7 y .265$ & 554.858 & 0.57 & 48678.88 \\
\hline
\end{tabular}

The following provides some numerical solutions for specific values of the parameters where $C=1 B=24 T=365$ $\delta=0.7$ while varying the values of $\beta$.

Table 1. Numerical solutions for specific values of the parameters while varying the values of $\beta$

$C=1 B=24 T \overline{=365 \quad \delta=0.7}$

Using the results of the table, above, we conclude that upon an increase in the parameter $\beta$ values, the length of each cycle is greater. As a result, the number of cycles decreases during $\bar{T}$ and the total value of active time at each cycle also increases. This also reflects another conclusion that when $\beta$ increases, both active time, $t_{A}$, and idle time, $t_{l_{n}}$ increase, until we approach an extreme optimal solution in which we see along $\bar{T}$ only one cycle i.e., $n \rightarrow 1$.

Example A2 (ii):

At Equation (10) we use the specific values of $\beta=\delta, \gamma=1$ and $\mathrm{A}=0$. 
In this case the idle time is also necessary for the process.

For the values above, Equation (10) is equal to:

$$
V M P_{L_{A}}=t_{I}^{\beta}\left(B-C t_{A}\right)
$$

As a result, the total value of revenue of each cycle, $v$, is:

$$
v=\frac{t_{\Sigma}^{\beta}\left(2 E-C t_{A}\right) t_{A}}{2}
$$

The number of cycles, $\mathrm{n}$, along period $\bar{T}$ is $n=\frac{\tau}{t_{A}+t_{I}}$.

Thus, $\mathrm{V}$ is the multiplication of $v \cdot n$ as follows:

$$
\left(M a x V=v \times n=\left(\frac{t_{I}^{\beta}\left(2 E-C t_{A}\right) t_{A}}{2}\right)\left(\frac{\bar{T}}{t_{A}+t_{I}}\right)\right.
$$

Taking the two derivatives of the F.O.C., with respect to $t_{A}$ and $t_{I}$ we can express $t_{A}$ in terms of $t_{I}$ as follows:

$$
t_{A}=\frac{E \beta t_{I}^{\beta}+C t_{I}^{\beta+1}-\sqrt{E^{2} t_{I}^{2 \beta} \beta^{2}+C^{2} t_{I}^{2(\beta+1)}}}{C t_{I}^{\beta} \beta}
$$

As shown, above, since we cannot solve the values analytically, we use specific values of the parameters while varying $\beta$ in the table below:

Here are some numerical examples for the specific values of $C=1 \quad T=365 B=24$ while varying $\beta$

Table 2. Numerical solutions for specific values of the parameters while varying the values of $\beta$

\begin{tabular}{llllll}
\hline$\beta$ & $t_{A}$ & \multicolumn{1}{c}{$t_{I}$} & $\mathrm{n}$ & \multicolumn{1}{c}{$\mathrm{V}$} \\
\hline 0.1 & 4.363 & 0.484 & 75.28 & 6666.75 \\
0.3 & 11.076 & 4.747 & 23.06 & 7526.47 \\
0.5 & 16 & 16 & 11.4 & 11680 \\
0.8 & 21.333 & 85.333 & 3.42 & 34131.59 \\
0.95 & 23.384 & 444.3 & 0.78 & 73576.5 \\
\hline
\end{tabular}

$C=1 \quad T=365 \quad B=24$

The results we get by maximizing the value $\mathrm{V}$ with respect to the two decision variables $t_{A}$ and $t_{I}$ that are derived simultaneously show that the optimal V obtained in Case 1 where $t_{I}$ is determined arbitrarily in advance for a specific value of $\beta=0.2$ and only $t_{A}$ was derived subject to a given $t_{I}$ is lower than the optimal $\mathrm{V}$ where $t_{A}$ and $t_{I}$ are determined simultaneously.

When $t_{I}$ and $t_{A}$ are determined simultaneously, $t_{A}=8, t_{I}=2, n=36.4, V=6708.398$

While the value of $\mathrm{V}$ when we assume arbitrarily that $\mathrm{t}_{\mathrm{l}}=1$ and then

$t_{A}=6, n=52.14 V$ is only equal to 6570

Which indicates as expected, an improvement of $\mathrm{V}$ by allowing simultaneous determination of $t_{A}$ and $t_{I}$ instead of imposing a given $t_{l}$.

A further example is shown below:

Example A2 (iii):

$\delta=0$ and $\mathrm{A}=0$

In this case, Equation (10) is equal to: $V M P_{t_{A}}=B t_{I}^{\beta}-C t_{A}^{\gamma}$ and the formula for $\mathrm{V}$ is the following:

$$
V=v \times n=\left(B t_{I}^{\beta} t_{A}-\frac{C}{\gamma+1} t_{A}^{\gamma+1}\right)\left(\frac{T}{t_{I}+t_{A}}\right)
$$

We continue this example with the further assumption $\gamma=1$, that means that the active time has linear impacts on the 
diminishing rate of the marginal productivity and then we get a new relationship between $t_{A}$ and $t_{f}$ follows:

$$
t_{A}=\frac{E t_{I}^{\beta}}{E t_{I}^{\beta}-1 \beta+C}
$$

Again, in the following table while assuming specific values of parameters $C=1 T=365 B=24$ we obtain the effect of changes in parameter $\beta$.

Table 3. Numerical solutions for specific values of the parameters while varying the values of $\beta$

\begin{tabular}{lllllc}
$\beta$ & $\tau_{A}$ & $t_{I}$ & $\mathrm{n}$ & $\mathrm{V}$ \\
\hline 0.1 & 4.53 & 0.57 & 71.54 & 6621.31 \\
0.2 & 12.84 & 4.28 & 21.32 & 7029.79 \\
0.3 & 39.93 & 29.94 & 5.22 & 9715.38 \\
\hline
\end{tabular}

$C=1 \quad T=365 \quad B=24$

In the table above, we conclude that upon increases in values of the parameter, the number of cycles decreases during $\bar{T}$ and the total value of both active time, $t_{A}$, and idle time, $t_{i}$, increases.

B) The Case in which Idle Time has a Constant Negative Effect on the Total Revenue

In the previous case $A$, above, idle time positively affects the active time, $t_{A}$, but has no direct positive or negative effect on revenue. In case $\mathrm{B}$, we assume that idle time has a negative effect on $\mathrm{V}$, by a constant rate for each additional unit of $t_{I}$. During active time the idle time has a positive influence, but a unit of idle time reduces the revenue by $\varepsilon$ dollars. Thus, after idle time of length $t_{I}$, there are cumulative expenses of, $\varepsilon t_{I}$, where $t_{I}$ is the length of the period of idle time.

The argument above reflects two different considerations. On one hand, idle time positively affects the consecutive active time, but on the other hand it creates some cost due to idling.

As a result, the total value of revenue of each cycle that is defined as , above, can be applied at (11) as follows:

$$
v=A t_{A}+B t_{I}^{\beta} t_{A}-\frac{c t_{t}^{\delta} t_{A}^{\gamma+1}}{\gamma+1}-\varepsilon t_{l}
$$

Since the total revenue of all cycles $V$ is defined as $v \cdot n$, we define the objective function of maximizing as follows:

$\operatorname{Max} V=v \times n=\left(A t_{A}+B t_{I}^{\beta} t_{A}-\frac{C t_{I}^{\delta} t_{A}^{\gamma+1}}{\gamma+1}-\varepsilon t_{L}\right)\left(\frac{\bar{T}}{t_{A}+t_{I}}\right)$

Example B1:

We repeat the examples shown in $\mathrm{A}$ in the new scenario of the cost of $\varepsilon$, similar to Example A1 when $\beta=1, \gamma=1, \delta=0$.

In such a case with the new value of $V>0$, the objective function is:

$$
V=\left[B t_{I} t_{A}+A t_{A}-\frac{C}{2} t_{A}^{2}-\varepsilon t_{I}\right]\left[\frac{\bar{T}}{t_{I}+t_{A}}\right]
$$

F.O.C. of each of the decision variables $t_{I}$ and $t_{A}$ solution is:

$$
t_{I}=\frac{C(A+\varepsilon)}{B\left(2 B^{+}+C\right)}
$$

and

$$
t_{A}=\frac{2(A+\varepsilon)}{2 E^{2}+C}
$$

Comparing the results of Example B1 to the results of Example A1, above, we see that in B1, the length of each period is greater than in Example A1. Thus, the number of cycles in Example B1 is smaller due to the idle time cost.

Example B2(i):

The same comparison is made with respect to Examples $\mathrm{B} 2$ and $\mathrm{A} 2$, above, when $\gamma=1$ and $\mathrm{A}=0$. We wish to maximize the value of $\mathrm{V}$ that is presented as follows: 


$$
V=\left(\frac{\left(2 E_{I}^{\beta}-C t_{I}^{\delta} t_{A}\right) t_{A}}{2}-\varepsilon t_{I}\right) \frac{\bar{T}}{t_{I}+t_{A}}
$$

Since there is no analytical solution, we take derivations with respect to both parameters, $t_{A}$ and $t_{I}$ and find at optimum the following:

$$
t_{A}=\frac{B \beta t_{I}^{\beta}+C t_{I}^{\delta+1}-\sqrt{E^{2} t_{I}^{2 \beta} \beta^{2}-2 B C t_{I}^{\delta+\beta+1} \delta+2 B C t_{I}^{\delta+\beta+1} \beta-2 C t_{I}^{\delta+1} \delta s+C^{2} t_{I}^{2(\delta+1)}}}{C t_{I}^{\delta} \delta}
$$

Since the analytical solution is impossible, we again use simulation presented in the table below.

Checking the same values as in Example $A 2(i)$ with $\varepsilon=2$ we obtain,

Table 4. Numerical solutions for specific values of the parameters while varying the values of $\beta$ and $\varepsilon=2$

\begin{tabular}{lllll}
\hline$\beta$ & $t_{A}$ & $t_{I}$ & $\mathrm{n}$ & \multicolumn{1}{c}{$\mathrm{V}$} \\
\hline 0.1 & 5.484 & 0.33 & 62.77 & 6919.8 \\
0.3 & 6.87 & 1.688 & 42.649 & 6632.133 \\
0.5 & 9.728 & 6.44 & 22.581 & 9150.5 \\
0.7 & 78.967 & 544.497 & 0.59 & 48040.74 \\
\hline
\end{tabular}

$C=1 \quad T=365 \quad B=24 \delta=0.7$

Comparing the results of Examples A2 and B2, we see that the number of cycles decreases as in the previous example.

Example B2 (ii):

$\beta=\delta, \quad \gamma=1$ and $\mathrm{A}=0$.

We seek a maximum for $\mathrm{V}$ as:

$$
V=\left(\frac{t_{I}^{\beta}\left(z B-C t_{A}\right) t t_{A}}{2}-\varepsilon t_{I}\right)\left(\frac{\bar{T}}{t_{I}+t_{A}}\right)
$$

We can find at optimum with respect to both parameters, $t_{A}$ and $t_{I}$ as follows:

$$
t_{A}=\frac{E \beta t_{l}^{\beta}+C t_{I}^{\beta+1}-\sqrt{B^{2} t_{I}^{2 \beta} \beta^{2}+2 C t_{l}^{\beta+1} \beta s+C^{2} t_{I}^{2(\beta+1)}}}{C t_{I}^{\beta} \beta}
$$

The same numerical examples as in Example A2 (ii), but with $\varepsilon=2$ now yield

Table 5. Numerical solutions for specific values of the parameters while varying the values of $\beta$ and $\varepsilon=2$

\begin{tabular}{lllll}
\hline \multicolumn{1}{c}{$\beta$} & \multicolumn{1}{c}{$\tau_{A}$} & $\tau_{l}$ & $\mathrm{n}$ & $\mathrm{V}$ \\
\hline 0.1 & 4.3636 & 0.436 & 76.04 & 6597.155 \\
0.3 & 11.076 & 4.316 & 23.711 & 7314.72 \\
0.5 & 16 & 15.03 & 11.76 & 11320.7 \\
0.8 & 21.333 & 83.516 & 3.48 & 33548.85 \\
\hline
\end{tabular}

$C=1 \quad T=365 \quad B=24$

We see that the number of cycles is almost the same as in the parallel Example A2(ii), with a very tiny increase.

Example B2 (iii):

$\gamma=1 \quad \delta=0$ and $\mathrm{A}=0$

We seek to maximize $\mathrm{V}$ as:

$$
V=\left(B t_{I}^{\beta} t_{A}-\frac{C}{\gamma+1} t_{A}^{\gamma+1}-\varepsilon t_{I}\right) \frac{\bar{T}}{t_{I}+t_{A}}
$$

We obtain the following relationship:

$$
t_{A}=\frac{t_{I}\left(B t_{I}^{\beta}+s\right)}{B t_{l}^{\beta} \beta+C t_{I}}
$$

When we take $C=1 \quad T=365 B=24 \gamma=1$ and $\varepsilon=2$ we obtain the following results: 
Table 6. Numerical solutions for specific values of the parameters while varying the values of $\beta$ and $\varepsilon=2$

\begin{tabular}{llllrl}
\hline$\beta$ & $\tau_{A}$ & $t_{I}$ & $\mathrm{n}$ & $\mathrm{V}$ \\
\hline 0.1 & 4.48 & 0.5 & 73.2 & 6543.96 \\
0.2 & 12.52 & 3.77 & 22.4 & 6854.16 \\
0.3 & 38.97 & 26.91 & 5.57 & 9409.19 \\
\hline
\end{tabular}

$C=1 \quad T=365 \quad B=24$

Comparing this case to the situation in which there is no cost, we see that in the latter the number of cycles is smaller.

\section{Conclusion}

The uniqueness of idle time has become an issue investigated in recent years by prominent scientists. It has a significant impact on the efficiency of the production process of goods and services, on learning and practicing and on various procedures like medical procedures. However, although idle time is required for efficient and effective utilization of time, it may often result in a negative effect on active time. It is of essential importance to analyze the interrelations between active and idle time and thus to control their combined effect on productivity, and time allocation.

The two different impacts should be considered.

On one hand, idle time positively affects the active time following it. Common examples are the importance of idle time for the routine activity of the brain during active time. So are working hours along the day that must include idleness and rest. Important and required idle times are due to personal needs such as coffee breaks, lunchtime, and relaxation time, all of which enable improved efficient active time. By the above factors, we may conclude that idle time is a constructive and important factor, required and helpful for using time efficiently and effectively.

On the other hand, however, idle time can also have a negative effect on active time that managers seek to eliminate or to minimize as much as possible. Common examples are performing required machinery adjustments, waiting between completing an old job and beginning a new one, waiting for instructions concerning a new task, or supplying material, tools and other preparations for forthcoming active time.

In this paper, we describe numerically these two types of idle and active times. We then try to give answers for how to maximize productivity taking into account the effects of active and idle time on each other and on the total net revenue.

The paper first presents the case in which idle time is determined a priory by law or by arbitrary decision makers. Then it is determined the optimal active time related to the given/ determined idle time. This optimization problem has an analytical solution depending on the parameters of the interdependent effect of idle time on the marginal productivity of active time. Then the case that at idle time and active time are both determined simultaneously is developed. However, in the first case there are no losses or costs of idle time on revenues.

As expected the results in terms of revenues and time allocation are better in the case of simultaneously determination in idle and active times. Then the case that during a constant marginal cost of idle time exists is discussed. Finally, the case where idle time marginal cost is increasing by a constant rate at each additional unit of idle time is discussed.

In the cases discussed above a positive intertemporal effect of idle time that positively affects the efficiency of active time in generating revenues is discussed but only with the case that idle time positively affects the active time.

The discussion adds several numerical examples since it is impossible to provide a precise analytical solution, yet sometimes we can analytically express the relationship between active time and idle time.

An important result that is shown in the cases is that upon an increase in the impacts of active time on the diminishing marginal productivity, the length of each cycle is greater. As a result, the number of cycles decreases during a certain time and the total value of active time at each cycle also increases. This also reflects another conclusion that both active time and idle time, increase.

In the second case, it is assumed that idle time has a negative effect on the revenue, by a constant rate at each additional until of idle time. During active time, the idle time has a positive influence, but the idle time itself may reduce directly the revenues due to shortening the active time. Thus, after idle time, there are cumulative expenses.

Comparing this case to the situation in which there is no revenue loss, it is shown as expected that in the latter case, the length of each cycle is greater and the number of cycles is smaller. 


\section{References}

Acharya, S. Control over Idle Time: Causes, Treatment and Specimen. http://www.yourarticlelibrary.com/cost-accounting/labour-cost/control-over-idle-time-causes-treatment-and-specim en $/ 57666$

Bloland, P. B., Ettling, M., \& Meek, S. (2000). Combination therapy for African malaria: hype or hope? Bull World Health Organ, 78(12), 1378-1388. http://www.who.int/bulletin/archives/78(12)1378.pdf

Bloland, P. B., Kazembe, P. N., Oloo, A. J., Himonga, B., Barat, L. M., \& Ruebush, T. K. (1998). Chloroquine in Africa: critical assessment and recommendations for monitoring and evaluating chloroquine therapy efficacy in sub-Saharan Africa. Trop Med Int Health, 3(7), 543-552. https://doi.org/10.1046/j.1365-3156.1998.00270.x

Bloland, P. B., Lackritz, E. M., Kazembe, P. N., Were, J. B., Steketee, R., \& Campbell. C. C. (1993). Beyond chloroquine: implications of drug resistance for evaluating malaria therapy efficacy and treatment policy. Africa. $J$ Infect Dis, 167(4), 932-937. https://doi.org/10.1093/infdis/167.4.932

Coleman, P. G., Morel, C., Shillcutt, S., Goodman, C., \& Mills, A. J. (2004). A threshold analysis of the cost-effectiveness of Artemisinin-based combination therapies in sub-saharan Africa. Am. J. Trop. Med. Hyg, 71(2), 196-204. https://www.ncbi.nlm.nih.gov/books/NBK3770/pdf/Bookshelf_NBK3770.pdf

Connolly, K. R., \& Thase, M. E. (2011). If at first you don't succeed: a review of the evidence for antidepressant augmentation, combination and switching strategies. Drugs, 71(1), 43-64. https://doi.org/10.2165/11587620-000000000-00000

Fredman, S. J., Fava, M., Kienke, A. S., White, C. N., Nierenberg, A. A., \& Rosenbaum, J. F. (2000). Partiak response, non-response, and relapse with selective serotonin reuptake inhibitors in major depression: a survey of current "next-step" practice. J Clin Psychiatry, 61(6), 403-408. https://doi.org/10.4088/JCP.v61n0602

Garcia-Toro, M. G., Roca, M., Monzón, S., Vives, M., Oliván, B., Vicens, E., Salva, J., \& Gili, M. (2012). Hygienic-dietary recommendations for major depression treatment: Study protocol of a randomized controlled trial. BMC Psychiatry, 12, 201. https://doi.org/10.1186/1471-244X-12-201

Goodman, C. A., Coleman, P. G., \& Mills, A. J. (2001). Changing the first line drug for malaria treatment cost-effectiveness analysis with highly uncertain inter-temporal trade-offs. Health Economics, 10(8), 731-749. https://doi.org/10.1002/hec.621

Graphics, Visualization and Usability (GVU) Centre, (1998) GVU's user surveys. Georgia Tech Research Corporation, October 1998, available at: http://WWW.cc.gatech.edu/gvu/user_surveys/.

Hirsch, M., \& Birnbaum, R. J. (2012). Antidepressant medication in adults: switching and discontinuing medication. In D. S. Basow (Ed.), UpToDate, Waltham, MA.

Innovation Tip: Schedule Idle Time, future think weblog - see https://futurethink.wordpress.com/2010/08/05/innovation-tip-schedule-idle-time

Lightner, N. J., Bose, I., \& Salvendy, G. (1996). What is wrong with the world-wide web? a diagnosis of some problems and prescription of some remedies. Ergonomics, 39(8), 995-1004. https://doi.org/10.1080/00140139608964523

Nedevschi, S., Chandrashekar, J., Liu, J., Nordman, B., Ratnasamy, S., \& Taft, N. (2009). Skilled in the Art of Being Idle: Reducing Energy Waste in Networked Systems. Proceeding NSDI'09 Proceedings of the $6^{\text {th }}$ USENIX symposium on Networked system design and implementation. 381-394. USENIX Association Berkeley, CA, USA 2009. USENIX Association Berkeley, CA, USA (C2009.

https://www.usenix.org/legacy/events/nsdi09/tech/full_papers/nedevschi/nedevschi_html/index.html

Ogle, N. R., \& Akkerman, S. R. (2013). Guidance for the discontinuation or switching of antidepressant therapies in adult. Journal of Pharmacy Practice, 26(4), 389-396. https://doi.org/10.1177/0897190012467210

Papakostas, G. I. (2009). Evidence for S-adenosyl-L-mentionine (SAM-e) for the treatment of major depressive disorder. Journal of Clinical Psychiatry, 70(5), 18-22. https://doi.org/10.4088/JCP.8157su1c.04

Pitkow, J. E., \& Kehoe, C. M. (1996). Emerging trends in the WWW user population. Communications of the ACM, 39(6), 106-108. https://doi.org/10.1145/228503.228525

Schapira, A., Beales, P. F., \& Halloran, M. E. (1993). Malaria living with drug-resistance. Parasitol Today, 9(5), 168-174. https://doi.org/10.1016/0169-4758(93)90140-B

Selvidge, P. (1999). How long is too long for a website to load? Usability News, 1(2). Available at: http://usabilitynews.org/how-long-is-too-long-to-wait-for-a-website-to-load 
Selvidge, P. (2003). Examining tolerance for online delays. Usability News, 5(1). Available at: $\mathrm{http} / /$ usabilitynews.org/examining-tolerance-for-online-delays

Souery, D., Serretti, A., \& Calati, R. (2011). Switching antidepressant class does not improve response or remission in treatment-resistant depression. J Clin Psycopharmacol, 31(4), 512-516. https://doi.org/10.1097/JCP.0b013e3182228619

Sudre, P., Breman, J. G., McFarland, D., \& Koplan, J. P. (1992). Treatment of chloroquine-resistant malaria in African children: a cost effectiveness analysis. Int J Epidemiol, 21(1), 146-154. https://doi.org/10.1093/ije/21.1.146

Swierniak, A., Kimmel, M., \& Smieja, J. (2009). Mathematical modelling as a tool for planning anticancer therapy. Eur. J. Pharmacol, 625, 108-121. https://doi.org/10.1016/j.ejphar.2009.08.041

Trivedi, R., Gupta, R. K., Husain, N., Rathore, R. K., Saksena, S., Srivastava, S., ... Narayana, P. A. (2009). Region-specific maturation of cerebral cortex in human fetal brain: diffusion tensor imaging and histology. Neuroradiology, 51(9), 567-576. https://doi.org/10.1007/s00234-009-0533-8

Ursrey, L. (2014). Your Brain Unplugged: Proof That Spacing Out Makes You More Effective. based on an interview with Andrew Smart, author of "Autopilot: The Art \& Science of Doing Nothing" http://www.forbes.com/sites/lawtonursrey/2014/05/16/your-brain-unplugged-proof-that-spacing-out-makes-you-m ore-effective/\#194e96986e 20

WHO/CTD. (1996). Assessment of Therapeutic Efficacy of Antimalarial Drugs for Uncomplicated Falciparum Malaria with Intense Transmission. Geneva: World Health Organization. WHO/MAL/96.1077.

Zietz, S., Desaire, C., Grattarola, M., \& Nicolini, C. (1978). Deterministic mathematical models to obtain optimized drug metabolism and cell kinetic parameters as a function of dosage from both autoradiographic and flow microffuorimetric analysis. In Biomathematics and Cell Kinetics (Ed.), A. J. Valleron, 431-438. Amsterdam: Elsevier.

\section{Copyrights}

Copyright for this article is retained by the author(s), with first publication rights granted to the journal.

This is an open-access article distributed under the terms and conditions of the Creative Commons Attribution license which permits unrestricted use, distribution, and reproduction in any medium, provided the original work is properly cited. 